\title{
Review: hippocampal volume is reduced in people with unipolar depression
}

Videbech P, Ravnkilde B. Hippocampal volume and depression: a meta-analysis of MRI studies. Am J Psychiatry 2004;161:1957-66.

$Q$ Is the volume of the hippocampus reduced in people with unipolar depression?

METHODS

Design: Systematic review with meta-analyses.

$\square$

Data sources: MEDLINE and EMBASE, plus hand searches of

reference lists; the electronic search covered the period 19662003.

Study selection and analysis: Studies reporting hippocampa volume in people with unipolar depression using magnetic resonance imaging (clinically diagnosed with DSM-IV, ICD-10, or equivalent) which used a control group without psychiatric disorders, neurological diseases, or drug/alcohol dependency. Other exclusion criteria were: control group much smaller than study group or use of a low resolution scanner. Studies in people with bipolar disorder were not meta-analysed. Meta-analyses used a random effects model and calculated the DersimonianLaird effect size. Heterogeneity was assessed using the $Q$ test and meta-regression, and publication bias was investigated with Begg's and Egger's tests. Calculations were performed with STATA v8.0.

Outcomes: Hippocampal volume $\left(\mathrm{mm}^{3}\right)$.

\section{MAIN RESULTS}

Twelve studies met inclusion criteria (351 people with depression and 279 healthy controls). Average hippocampal volume was reduced by $8 \%$ in the left side and $10 \%$ on the right side in people with unipolar depression (standardised mean difference in hippocampal volume between people with depression and controls: left side -0.38 , $95 \%$ CI -0.65 to -0.11 ; right side $-0.32,95 \%$ CI -0.56 to -0.08 ). There was significant heterogeneity between studies $(\mathrm{p}<0.003$ left side; $\mathrm{p}<0.01$ right side) but there was no evidence of publication bias $(\mathrm{p}=0.135)$

\section{CONCLUSIONS}

Hippocampal volume is reduced in people with unipolar depression.

\section{Commentary}

V idebech and Ravnkilde's meta-analysis has identified a significant association between major depressive disorder and bilateral volume reduction of the hippocampus. It is a hypothesis driven review that used systematic selection criteria of empirical studies, as well as stringent statistical methods (for example, analyses assuring that no single study skewed the results). In addition, the absence of evidence for publication bias lends further credence to the validity of the findings.

The considerable heterogeneity identified suggests that hippocampal volume reduction may be present in some but not all people with depression, or at some but not all stages of illness. Thus, studies of recurrent depression demonstrated larger reductions of right hippocampus volumes than studies of people with first episode depression. It will be important to conduct further research with more homogeneous samples in order to identify other clinical moderators of hippocampal volumes in depression (for example, early adverse experiences, comorbidity). Other possible sources of heterogeneity include differences in study design and imaging methodology that can affect sensitivity to variation in hippocampus volumes: sample size, acquisition sequences, slice thickness, and the definition and reliability of anatomical delineations. ${ }^{1-3}$ Unfortunately, the authors did not examine whether any of these parameters contributed to within or between group variation in hippocampal volume. Such analyses would be useful to guide the design of future MRI studies of depression.

It is too early to conclusively establish the timing of hippocampal volume loss in major depression. One possibility is that it predates onset of affective symptoms, and thereby represents a biological risk marker. Thus far, cross-sectional studies largely have not found evidence of hippocampal atrophy in first episode or paediatric depression. ${ }^{4-7}$ Instead, this meta-analysis is more compatible with hypotheses that hippocampal atrophy arises from a neurotoxic progression of the (untreated) disease. ${ }^{89}$ This underlines the importance of intervening clinically at the first signs of acute depression, and of maintaining an aggressive treatment regimen during periods of partial or complete remission. Even so, we await the results of longitudinal studies of high risk subjects and homogeneous samples to refine our treatment targets and interventions.

Dr Isabelle M Rosso, PhD, Instructor Department of Psychiatry, McLean Hospital and Harvard Medical School,

1 Brierley B, Shaw P, David AS. The human amygdala: a systematic review and meta-analysis of volumetric magnetic resonance imaging. Brain Res Rev 2002;39:84-105.

2 Campbell S, Marriott M, Nahmias C, et al. Lower hippocampal volume in patients suffering from depression: a meta-analysis. Am J Psychiatry 2004;161:598-607.

3 Sheline YI. 3D MRI studies of neuroanatomic changes in unipolar major depression: the role of stress and medical comorbidity. Biol Psychiatry 2000;48:791-800

4 MacMaster FP, Kusumakar V. Hippocampal volume in early onset depression. BMC Med 2004;2:2.

5 MacMillan S, Szeszko PR, Moore GJ, et al. Increased amygdala: hippocampal volume ratios associated with severity of anxiety in pediatric major depression. J Child Adolesc Psychopharmacol 2003;13:65-73.

6 MacQueen GM, Campbell S, McEwen BS, et al. Course of illness,

hippocampal function, and hippocampal volume in major depression. Proc Natl Acad Sci USA 2003;100:1387-92.

7 Rosso IM, Cintron CM, Steingard RJ, et al. Amygdala and hippocampus volumes in pediatric major depression. Biol Psychiatry 2005;57:21-6.

8 McEwen BS. Mood disorders and allostatic load. Biol Psychiatry 2003:54:200-7.

9 Sapolsky RM. The possibility of neurotoxicity in the hippocampus in major depression: a primer on neuron death. Biol Psychiatry 2000;48:755-65. 